\title{
Implementation of an Inhaled Nitric Oxide Protocol: A Paradox or the Perfect Pair?
}

Nitric oxide was first described over 200 years ago, initially for its toxic effects. ${ }^{1}$ More recently, nitric oxide was named Molecule of the Year in 1992 by the journal Science. In 1998, the Nobel Prize in Physiology and Medicine was awarded to 3 scientists for further elucidating nitric oxide's signaling role in the cardiovascular and nervous systems. ${ }^{2} \mathrm{~A}$ pivotal randomized controlled trial by the Neonatal Inhaled Nitric Oxide Study Group was published in 1997 and was instrumental to the 1999 FDA clearance of the use of inhaled nitric oxide (INO) in term or near-term infants with hypoxic respiratory failure. ${ }^{3}$ This work started the discussion regarding cost associated with INO delivery because the implementation of it, although expensive, reduced the need for other more expensive resources, including extracorporeal membrane oxygenation. INO in preterm animal models has demonstrated a reduction in pulmonary vascular resistance, improved surfactant function, stimulated angiogenesis, and enhanced alveolar formation. ${ }^{4-7}$ Based on this evidence, the hope was that INO would reduce right ventricular failure as well as the need for prolonged respiratory support with potentially toxic oxygen levels, leading to a reduction in ventilator-induced lung injury. However, randomized controlled trials in preterm infants have remained largely negative, and the use of INO to prevent bronchopulmonary dysplasia is still not recommended. 8,9

Despite considerable effort, there is a paucity of large, randomized, double-blinded, multi-center clinical trials for pediatric hypoxic respiratory failure. Although it is clear that INO transiently improves arterial oxygenation, rarely does the evidence demonstrate a sustained benefit. ${ }^{10}$ In current practice, INO is still prescribed for some pediatric hypoxic respiratory failure despite high cost and unconvincing evidence. This is partially due to the belief that those who benefit are the severely hypoxic, who are thought to be an underrepresented cohort in clinical trials. That said, based on current clinical evidence, INO cannot be routinely recommended for pediatric hypoxic respiratory failure. ${ }^{10}$

\footnotetext{
The authors have disclosed no conflicts of interest.
}

Correspondence: Brian K Walsh RRT-NPS FAARC, Boston Children's Hospital, Office LO254, 333 Longwood Avenue, Boston, MA 02115. E-mail: brian.walsh@childrens.harvard.edu.

DOI: $10.4187 /$ respcare. 04074
Surgical interventions for congenital heart disease can be complicated by pulmonary hypertension. Proper management of postoperative pulmonary hypertension can reduce mortality, morbidity, and medical burden. ${ }^{11}$ INO has been suggested to have a physiologic benefit in these subjects in preclinical and small uncontrolled trials. ${ }^{12,13}$ INO is often

See the Original Study on Page 644

prescribed to treat pulmonary hypertension in the cardiac ICU setting; however, its overall effectiveness in improving outcomes and in comparison with other available pulmonary vasodilators has yet to be determined. In addition, Simsic et $\mathrm{al}^{14}$ reported that the use of an INO protocol in this subject population reduced variation, but not utilization or cost. This is in contrast to a study in this issue of RESPIRATORY CARE.

In this issue of Respiratory CARE, Todd Tzanetos et al ${ }^{15}$ describe the implementation of an INO protocol and its associated cost reduction in a retrospective cohort of 76 pediatric subjects. Their goal was to determine whether implementation of an INO protocol would reduce cost of delivery. This study successfully builds upon the results of previous findings that protocolized care delivered by respiratory therapists can be a more efficient means of providing evidence-based therapy. ${ }^{16-24}$ The authors conclude that the protocol reduced costs without significantly differing mortality. However, it remains unclear if the protocol really reduced the cost of care.

As mentioned in the introduction, the hospital cost of INO was $\$ 100 / \mathrm{h}$ during the review period. The median change in INO duration trended toward significance, but was not statically significant. This implies that the cost was based on patient billing, not cost of delivery. Likewise, the hospital was not reimbursed what it cost to deliver INO, as the median cost divided by the median duration was $\$ 76.5$ pre-protocol and $\$ 91$ post-protocol. Insurers may contractually withhold full reimbursement for expensive therapies that have not been proven to be effective in an effort to discourage the practice. It appears that as the protocol reduced the duration of INO, the cost to the subject increased per $h$. If expense to the hospital had been calculated based on a rate of $\$ 100 / \mathrm{h}$, the median reduction in hospital expense would have been more pronounced.

Many institutions providing neonatal and pediatric care are spending in excess of a million dollars per year on INO. The 
most efficient way to reduce the cost of expensive and unproven therapies is not to start them. Although the duration of INO was not statically significant, the most beneficial section of the protocol was the quick discontinuation for nonresponders. Many hospitals serving primarily adults have eliminated INO from their practice, reserved INO for a very select group with special oversight, or switched to alternative vasodilators in an effort to reduce the cost of an unproven therapy. ${ }^{25-27}$ Proponents of nitric oxide argue that the current evidence is based on the wrong patient populations. This may be valid, but given the cost of treatment and the marginal benefits on arterial oxygenation, it is unlikely that a clinical trial will be performed with sufficient sample size or patient population to address these concerns.

This study leads us to a paradox. Should we protocolize the use of INO when there is insufficient evidence for its therapeutic benefit? It is well understood that the current means of resolving nonbeneficial treatment are inadequate. ${ }^{28}$ Or are INO and a well-developed protocol the perfect pair? Could strict protocolization of questionable therapies be an interim technique used to provide the therapy in a more costeffective way until more evidence is gathered? Although Todd Tzanetos et al ${ }^{15}$ do not condone the use of INO, they logically implemented a protocol to reduce practice variance in an effort to reduce cost, and they must be applauded. The attractive components of this protocol are the staff educational methods, the targets of a therapeutic response, and the raised awareness of therapy costs. It is possible that this protocol could continue to be refined to meet a variety of patient needs, with the least amount of resources. However, the ideal model for protocols is likely one that incorporates evidencebased guidelines. In that regard, INO outside of the neonatal arena still remains controversial.

\section{Brian K Walsh RRT-NPS FAARC Jordan S Rettig MD Division of Critical Care Medicine Department of Anesthesia Boston Children's Hospital Boston, Massachusetts}

\section{REFERENCES}

1. Priestley J. Experiments and observations on different kinds of air, volume II. London: J. Johnson; 1775.

2. SoRelle R. Nobel prize awarded to scientists for nitric oxide discoveries. Circulation 1998;98(22):2365-2366.

3. Neonatal Inhaled Nitric Oxide Study Group. Inhaled nitric oxide in full-term and nearly full-term infants with hypoxic respiratory failure. N Engl J Med 1997;336(9):597-604.

4. Lin YJ, Markham NE, Balasubramaniam V, Tang JR, Maxey A, Kinsella JP, Abman SH. Inhaled nitric oxide enhances distal lung growth after exposure to hyperoxia in neonatal rats. Pediatr Res 2005;58(1):22-29.

5. Ballard PL, Gonzales LW, Godinez RI, Godinez MH, Savani RC, McCurnin DC, et al. Surfactant composition and function in a pri- mate model of infant chronic lung disease: effects of inhaled nitric oxide. Pediatr Res 2006;59(1):157-162.

6. Bland RD, Albertine KH, Carlton DP, MacRitchie AJ. Inhaled nitric oxide effects on lung structure and function in chronically ventilated preterm lambs. Am J Respir Crit Care Med 2005;172(7):899-906.

7. Tang JR, Markham NE, Lin YJ, McMurtry IF, Maxey A, Kinsella JP, Abman SH. Inhaled nitric oxide attenuates pulmonary hypertension and improves lung growth in infant rats after neonatal treatment with a VEGF receptor inhibitor. Am J Physiol Lung Cell Mol Physiol 2004;287(2):L344-L351.

8. Cole FS, Alleyne C, Barks JD, Boyle RJ, Carroll JL, Dokken D, et al. NIH Consensus Development Conference statement: inhaled nitricoxide therapy for premature infants. Pediatrics 2011;127(2):363-369.

9. Jensen EA, Kirpalani H. Inhaled nitric oxide to prevent bronchopulmonary dysplasia in preterm infants-less than a silver bullet. J Pediatr 2014;165(6):1079-1081.

10. Afshari A, Brok J, Møller AM, Wetterslev J. Inhaled nitric oxide for acute respiratory distress syndrome and acute lung injury in adults and children: a systematic review with meta-analysis and trial sequential analysis. Anesth Analg 2011;112(6):1411-1421.

11. Checchia PA, Bronicki RA, Goldstein B. Review of inhaled nitric oxide in the pediatric cardiac surgery setting. Pediatr Cardiol 2012; 33(4):493-505.

12. Kawakami H, Ichinose F. Inhaled nitric oxide in pediatric cardiac surgery. Int Anesthesiol Clin 2004;42(4):93-100.

13. Ichinose F, Roberts JD Jr, Zapol WM. Inhaled nitric oxide: a selective pulmonary vasodilator: current uses and therapeutic potential. Circulation 2004;109(25):3106-3111.

14. Simsic JM, Harrison S, Evans L, McClead R, Teske D, Institute of Healthcare Improvement. Reducing variation in the use of inhaled nitric oxide. Pediatrics 2014;133(6):e1753-e1758.

15. Todd Tzanetos DR, Housley JJ, Barr, FE, May, WL, Landers, CD. Implementation of an inhaled nitric oxide protocol decreases direct cost associated with its use. Respir Care 2015;60(5):644-650.

16. Berg M. Problems and promises of the protocol. Soc Sci Med 1997; 44(8):1081-1088.

17. Maselli DJ, Fernandez JF. Can respiratory therapist-driven protocols improve resource utilization? Respir Care 2013;58(3):546-547.

18. Chatburn RL, Deem S. Respiratory controversies in the critical care setting. Should weaning protocols be used with all patients who receive mechanical ventilation? Respir Care 2007;52(5):609-619; discussion 619-621.

19. Stoller JK. The effectiveness of respiratory care protocols. Respir Care 2004;49(7):761-765.

20. Kollef MH, Shapiro SD, Clinkscale D, Cracchiolo L, Clayton D, Wilner R, Hossin L. The effect of respiratory therapist-initiated treatment protocols on patient outcomes and resource utilization. Chest 2000;117(2):467-475.

21. Stoller JK, Kester L. Respiratory care protocols in postanesthesia care. J Perianesth Nurs 1998;13(6):349-358.

22. Zimmerman L. Respiratory therapist-driven protocols. West J Med 1997;167(6):440-441.

23. Stoller JK. Respiratory therapist-driven protocols. Rationale and efficacy. West J Med 1997;167(6):408-410.

24. Burton GG. A short history of therapist-driven respiratory care protocols. Respir Care Clin N Am 1996;2(1):15-26.

25. Siobal MS, Hess DR. Are inhaled vasodilators useful in acute lung injury and acute respiratory distress syndrome? Respir Care 2010; 55(2):144-157; discussion 157-161.

26. Siobal MS. Pulmonary vasodilators. Respir Care 2007;52(7):885-899.

27. Siobal M. Aerosolized prostacyclins. Respir Care 2004;49(6):640-652.

28. Downar J, You JJ, Bagshaw SM, Golan E, Lamontagne F, Burns K, et al. Nonbeneficial treatment Canada: definitions, causes, and potential solutions from the perspective of healthcare practitioners. Critical Care Med 2015;43(2):270-281. 